\title{
A Platform for Health Record Management of the Conscripts in the Hellenic Navy
}

\author{
Olympia GIANNAKOPOULOU ${ }^{\mathrm{a}}$, Petros TOUMPANIARIS ${ }^{\mathrm{a}, 1}$, Ioannis KOURIS ${ }^{\mathrm{a}}$, \\ Konstantia MOIROGIORGOU ${ }^{\mathrm{b}}$, Nansy KARANASIOU ${ }^{\mathrm{c}}$, Vasiliki AISOPOU ${ }^{\mathrm{d}}$, \\ George MATSOPOULOS ${ }^{\mathrm{a}}$, Ageliki CHANDRINOUd, \\ Nikolaos ANOUSAKIS - VLACHOCHRISTOU ${ }^{\mathrm{d}}$, Fotis PSARROS ${ }^{\mathrm{d}}$, \\ Pantelis SYRIGAS ${ }^{a}$, Vasiliki KARALIDOU ${ }^{a}$, Nikolaos COSTARIDIS ${ }^{\mathrm{e}}$, \\ Michalis ZERVAKIS ${ }^{\mathrm{b}}$ and Dimitris KOUTSOURIS ${ }^{\mathrm{a}}$ \\ ${ }^{a}$ Institute of Communications and Computer Systems, \\ National Technical University of Athens, Greece \\ ${ }^{\mathrm{b}}$ Technical University of Crete, Greece \\ ${ }^{c}$ Vidavo S.A., Greece \\ ${ }^{d}$ Naval Hospital of Athens, Greece \\ ${ }^{e}$ Dpt. for the Management of European \& Developmental Projects, \\ Ministry of Greek National Defence, Greece
}

\begin{abstract}
Mass project aims to digitalize the medical examination procedure of recruitment phase of conscripts in the Hellenic Navy. eMass integrates recruits' Electronic Health Record (EHR), while allows a pre-screening test, through portable telemedicine equipment. The data will be exploited to assess the individual's cardiovascular risk through appropriate digital tools and algorithms. The eMass digital platform, will be accessible to health experts involved in the recruitment procedure for further assessment and processing. Recruits' personal data is stored in the database encrypted using Advanced Encryption Standard (AES). eMass solution contributes to beneficial management and medical data analysis, preventing inessential physical or medical examinations minimizing danger of possible errors and reducing time-consuming processes. Moreover, eMass exploits Electronic Health Record data through a machine-learning based cardiovascular risk assessment tool.
\end{abstract}

Keywords. Armed Forces, medical examination procedure, recruits EHR

\section{Introduction}

Nowadays, in Greece, males from 18 to 45 years old with Greek citizenship are obliged to serve in the Hellenic Armed Forces. The training divisions are conducted four to six times annually and the average amount of recruits in the Hellenic Navy at each of them varies from 400 to $1,000[1]$. For each recruit a standard and prespecified medical examination is performed, involving a variety of medical specialties. So far, the medical examination procedure is executed in the absence of any kind of digitalization. This weakness results in lack in the follow up, disorganization of both the human resources assets and causes difficulties in standardized recordings and control.

\footnotetext{
${ }^{1}$ Corresponding Author, Petros Toumpaniaris; E-mail: petros.toumpaniaris@gmail.com.
} 


\section{2. eMass Platform}

eMass project develops a platform that expedites the medical examination procedure of recruitment process in the Hellenic Navy. The platform integrates recruits' Electronic Health Record (EHR) by extracting appropriate health indicators, which are considered as a significant key factor for actions towards recruits' medical assessment [2].

Specifically, an EHR will be conducted for each recruit by the staff of the army classification unit, in which, recruit's demographics, personal and family medical history will be stored. Moreover, a pre-screening test, through portable telemedicine equipment collecting ECG, blood pressure and oximetry signals, is scheduled to be performed. The data will be stored in the recruit's EHR and will be exploited to estimate the individual's cardiovascular risk through appropriate digital tools and algorithms. The eMass digital platform, including all the stored data, will be accessible to health experts of both local and central military medical units, for further assessment and processing, enabling them to access recruits' EHRs and advanced health data processing solutions.

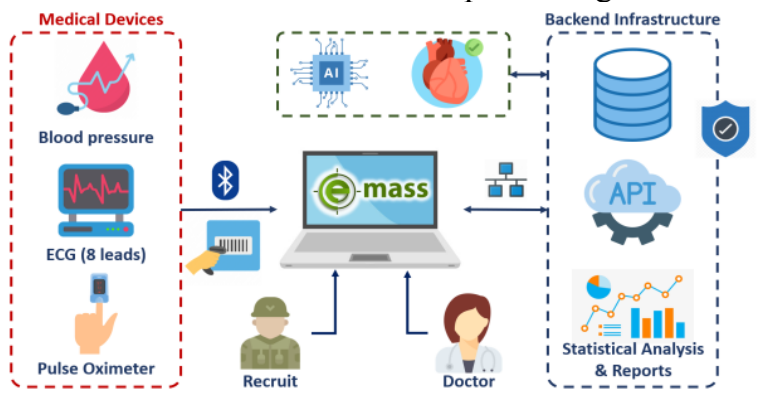

Figure 1. eMass platform.

\section{3. eMass Data Flow \& Privacy Mechanism}

The data flow in eMass platform is described by each recruit's medical examination during his military recruitment process. The process is described using the steps below: (i) Every army recruit visits a military entrance processing station. (ii) The army staff search his medical record using the conscription or social security number. As soon as the recruit has been successfully identified, his primary personal information will be displayed (i.e. Name, Surname, Father's / Mother's name, and social security number). (iii) The recruit's status is directly updated to "PRESENTED". (iv) A single QR code is then provided and printed for the recruit. The recruit is then forwarded to a specially designed area where all the necessary medical staff is located, including eight medical specialties (pathology, cardiology, radiology, otorhinolaryngology, microbiology ophthalmology, surgery - orthopedics, and neurology - psychiatric). Each one of the recruits visits each medical specialty's office, where the single code that is provided at the entrance point is scanned and the appropriate data is entered. Recruits' personal data is stored in the database encrypted using Advanced Encryption Standard (AES). AES is a symmetric - key block cipher type of cryptography method. It consists of two processes, the encryption process and the decryption process. Each of them has six steps. The decryption process is performed by reversing the steps of the encryption process. There are three variants of AES: AES-128, AES-192 and AES-256 [3]. 


\section{4. eMass Technical \& Technological Characteristics}

The final eMass system is composed of the following cooperating subsystems: (i) Telemedicine subsystem, (ii) barcode/QR code operating and printing subsystem, (iii) EHR subsystem, (iv) Data analysis for cardiovascular diseases prediction, (v) Backend services/APIs.

\subsection{Telemedicine Subsystem}

Telemedicine Subsystem consists of: (i) software interconnection of portable medical devices that will be used, (ii) user interfaces, (iii) interoperability with backend services/APIs. Based on the required medical examinations eMass telemedicine subsystem uses the following medical devices: (i) 12-lead electrocardiograph with Bluetooth, (ii) sphygmomanometer with Bluetooth, (iii) oximeter with Bluetooth. Interconnection software development for each one of the devices is required in order to be integrated in eMASS system. Considering that telemedicine devices use BLE protocol, but EHR application is a web-based application APIs is the only possible solution for the subsystems' integration. The connection with the telemedicine application in Windows 10 occurs through GATT protocol.

\subsection{Barcode/QR code operating and printing subsystem}

Barcode/QR code operating and printing subsystem supports NHA requirement for the provision of a barcode/QR code for each recruit at the hospital's Gate in to allow their proper identification during the whole recruitment process. QR code provides high data storage capacity, fast scanning, omnidirectional readability, and many other advantages including, error-correction and different type of versions[4]. This requirement leads to an API integrated subsystem, enabling identification data storage, single code creation, and printer connection.

\subsection{EHR subsystem}

EHR subsystem is accessible through web browser and contains significant and various health data. The purpose of the EHR is to record the diagnoses, the medical history and in general the previous and the current medical condition of the conscripts [5]. The aim is to support, digitize the classification process and the health file to focus on the health examination and the data collected during the classification. In this way, each new recruit is able to be evaluated both clinically at the local level, and through modern technological means and interconnection with a central unit of control and case management.

\subsection{Data analysis for cardiovascular diseases prediction}

Expert systems (ES) constitute one of the new, promising trends in data analysis field. They are designed to perform in advising, decision making, diagnosing, and prediction[6]. eMass expert system is aimed to help NHA doctors using medical data recorded in eMass platform and contribute in decision making for cardiovascular risk assessment. eMass expert system contributes in control and record of two relatively often heritable cardiovascular diseases in males: (i) bicuspid aortic valve, and (ii) atrial septal 
defect. Consequently, echordiaography will be performed in a large scale, aiming to be performed at least for 1000 recruits. The purpose of this tactic is to define a methodology for the number of individuals as the best control option by validating it, using statistical terms where needed. In particular, the eMass Expert System consists of 2 subsystems: (i) Echocardiography Image and Video Analysis Subsystem, (ii) Laboratory Examinations and Medical History Analysis for Cardiovascular Function Assessment Subsystem.

\subsection{Backend services/APIs}

Backend Subsystem consists of a set of software and tools, located in one server in NHA. In particular backend consists of (i) relational database which stores all the data per recruit, (ii) API, (iii) the software that serves system's functions, (iv) echocardiography data analysis. The Relational model is now the dominant model in almost all database implementations. It consists of a collection of logical records grouped into relationships (normalized tables), which have unique names and attributes (fields). Connections are only achieved through their common connection fields with a line representing a one-toone correlation and so each table represents a collection of one-to-one correlations [7].

\section{5. eMass Use Cases}

\subsection{Use case 1}

Recruit A.B. arrives at the military recruiting station, where the admissions office records the details of the recruit based on the greek Social Insurance Registration Number, or the particular details are being confirmed in case that an appropriate csv which includes all the recruits' details has already been loaded. Then, a single code is being provided to the recruit, based on which he is enabled to continue the recruitment process by visiting the doctors' offices (in a random manner). In each of them, recruit's medical history, clinical examination as well as other details are recorded to recruit's EHR. At the end of the procedure recruit A.B. returns at the admissions office, where the overall record with the final decision for the physical ability classification is printed and signed by the recruit.

\subsection{Use case 2}

Recruit A.C arrives at the military recruiting station and during his visit in cardiologist's office a serious cardiological issue is detected. The cardiologist begins the medical referral procedure by recording the medical findings on his computer. The recruit passes through the rest of the doctors' offices and then returns at the admissions office, where he receives the referral and signs the documents waiting to be transferred to the NHA for further examinations. NHA's medical staff has read-only access to the final referral. The Cardiological Clinic's Manager and the doctor in charge consider the recruit's medical conditions and classify him in class 4 regarding his physical ability. At the end of the procedure recruit A.B. returns at the military recruiting station, provides the necessary documents and the final decision is made. The administration updates the recruit's file with his final decision through the suitable interface and the file is finally closed. 


\section{Conclusions}

eMass development and implementation capitalizes on constant detection of health status and medical history for each recruit, contributing to agile and accurate diagnosis of medical issues. Efficient management of medical examination scheduling as the digitalization leads to automated processes, following a defined flow, without deviations contributes in beneficial management and medical data analysis, preventing repeating inessential physical or medical examinations and, consequently, minimizing danger of possible errors [8] [9]. Although the delay is not decreased, however this is done for the benefit of the quality which increases significantly. eMass reinforces the enhancement and establishment in the overall process of medical data collection and recording. Moreover, additional cases are identified for further examination and the conscripts are benefited from a thorough examination [2]. The exploitation of a recruiter's EHR through the deployment of the machine-learning based cardiovascular risk assessment tool is one of the notable eMass assets. This information provided is recorded in the recruit's EHR and supports medical experts in critical decisions [10]. Since the algorithm is trained only by using medical data of male subjects, has limitations on its use in the general population. As a future work this algorithm should be generalized and enhanced using data collected from female subjects.

\section{Acknowledgment}

This research has been co - financed by the European Union and Greek national funds through the Operational Program Competitiveness, Entrepreneurship and Innovation, under the call RESEARCH - CREATE - INNOVATE (project code:T1EDK-03505)

\section{References}

[1] "Hellenic Army General Staff | Army gr.".

[2] Pohlmann S, et al. Digitalizing health services by implementing a personal electronic health record in Germany: Qualitative analysis of fundamental prerequisites from the perspective of selected experts. J. Med. Internet Res. 2020;22(1).

[3] Ratnadewi R, Adhie RP, Hutama Y, Christian J, Wijaya D. Implementation and performance analysis of AES-128 cryptography method in an NFC-based communication system. World Transactions on Engineering and Technology Education. 2017;15(2).

[4] Tiwari S. An Introduction to QR Code Technology. 2017:39-44.

[5] Mihailescu M, Mihailescu D, Carlsson S. Understanding Healthcare Digitalization: A Critical Realist Approach. ICIS 2017 Proc., Dec. 2017.

[6] Clancey WJ. The epistemology of a rule-based expert system -a framework for explanation. Artif. Intell. 1983;20(3):215-251.

[7] Bressoud T, White D. Relational Model: Design, Constraints, and Creation, in Introduction to Data Systems, Cham: Springer International Publishing, 2020: 425-462.

[8] Hoover R. Benefits of using an electronic health record. Nurs. Crit. Care. 2017;12(1):9-10.

[9] Bowman S. Impact of Electronic Health Record Systems on Information Integrity: Quality and Safety Implications. American Health Information Management Association. 2013;10.

[10] Sacramento-Pacheco J, et al. Cardiovascular risk assessment tools: A scoping review. Australian Critical Care. 2019;32(6):540-559. 